\title{
NEUer at SemEval-2021 Task 4: Complete Summary Representation by Filling Answers into Question for Matching Reading Comprehension
}

\author{
Zhixiang Chen ${ }^{1 \dagger}$, Yikun Lei ${ }^{1 \dagger}$, Pai Liu ${ }^{1,2 \dagger}$, Guibing Guo ${ }^{1^{*}}$ \\ ${ }^{1}$ Northeastern University, Shenyang, China \\ ${ }^{2}$ Westlake University, Hangzhou, China \\ $\dagger$ These three authors contributed equally. \\ \{zxchen8830, ethanlei9931, pailiul998\}@gmail.com \\ guogbeswc.neu.edu.cn
}

\begin{abstract}
SemEval task 4 aims to find a proper option from multiple candidates to resolve the task of machine reading comprehension. Most existing approaches propose to concat question and option together to form a context-aware model. However, we argue that straightforward concatenation can only provide a coarsegrained context for the MRC task, ignoring the specific positions of the option relative to the question. In this paper, we propose a novel MRC model by filling options into the question to produce a fine-grained context (defined as summary) which can better reveal the relationship between option and question. We conduct a series of experiments on the given dataset, and the results show that our approach outperforms other counterparts to a large extent.
\end{abstract}

\section{Introduction}

In order to make the computer understand, represent and express better, we study the ability of MRC to understand Abstract definitions(Spreen and Schulz, 1966; Changizi, 2008) in the Reading Comprehension of Abstract Meaning task(ReCAM). In ReCAM task, there are two kinds of abstract definitions, one is imperceptibility and the other is nonspecificity. To evaluate the model performance comprehensively, three subtasks are designed(Zheng et al., 2021):

Subtask 1) ReCAM-Imperceptibility - A passage, a question with a placeholder, and answers used to fill in the placeholder are given. The answers are all imperceptibility words so as to evaluatethe model's ability to comprehend these imperceptibility words.

Subtask 2) ReCAM-Nonspecificity - A passage, a question with a placeholder, and answers used to fill in the placeholder are given. The answers are all Nonspecificity words so as to evaluatethe model's ability to comprehend these Nonspecificity words.

Subtask 3) To provide more insights into the relationship between two abstract views, it requires to test the performance of the model trained on one data set and evaluated on the other one.

Because question is obtained by hollowing out summary of passage and answer is abstract words to fill in(Zheng et al., 2021), there is a semantically complementary relationship between the question and answer. As shown in the Figure 1, in the previous work(Jin et al., 2020; Zhu et al., 2020), the model inputs passage, question and answer independently. It can only provide a coarsegrained context for the MRC task, ignoring the specific positions of the option relative to the question. Quesiton cannot be a complete sentence, and the answer lacks contextual information about the question. To solve this problem, we introduce Complete Summary Representation by Filling Answers into Question for Maching Reading Comprehension(ComSR). ComSR convert the original question and answer into summary. In this way, we can turn the problem into matching correct summary for the passage. By replacing the answer option into the question placeholder, the original question has higher semantic integrity, and the original answer obtains the context information from the question to eliminate language ambiguity such as polysemy.

Based on the results, ComSR is superior to pretrained language models and the latest MRQA models, with the accuracy rate of $64.76 \%$ in subtask 1 , $64.86 \%$ in subtask 2, and shows great generalization ability in Subtask 3.

\section{System overview}

Figure 2 illustrates the overall structure of ComSR. Since just one summary option belongs to the pas- 


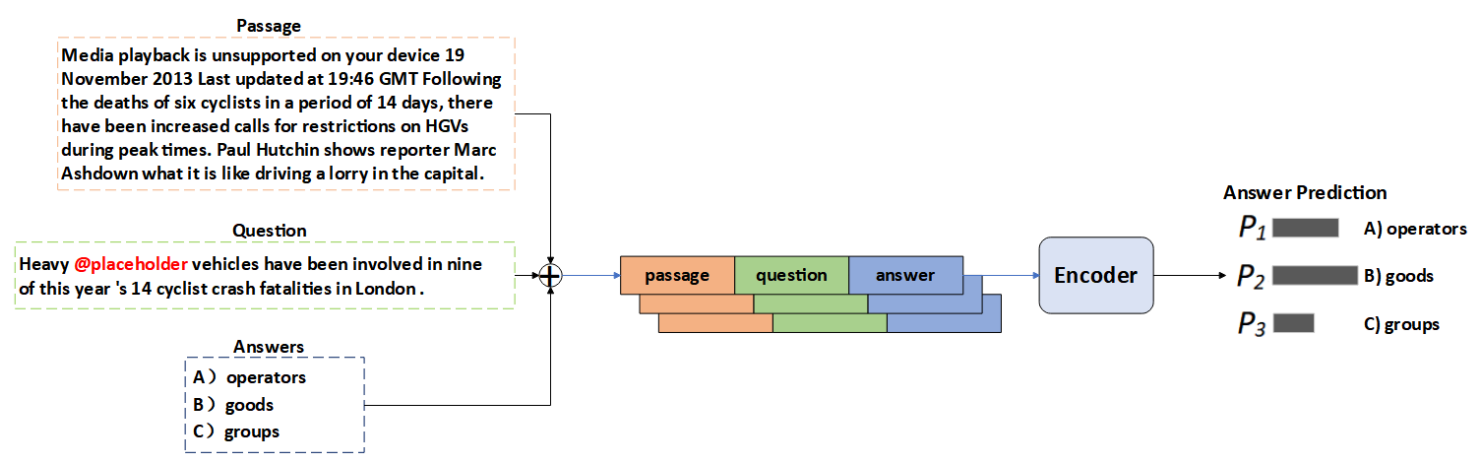

Figure 1: The framework of previous MCQA model. The abstract word in answer has a polysemy phenomenon. Entering question and answer separately cannot eliminate ambiguity.

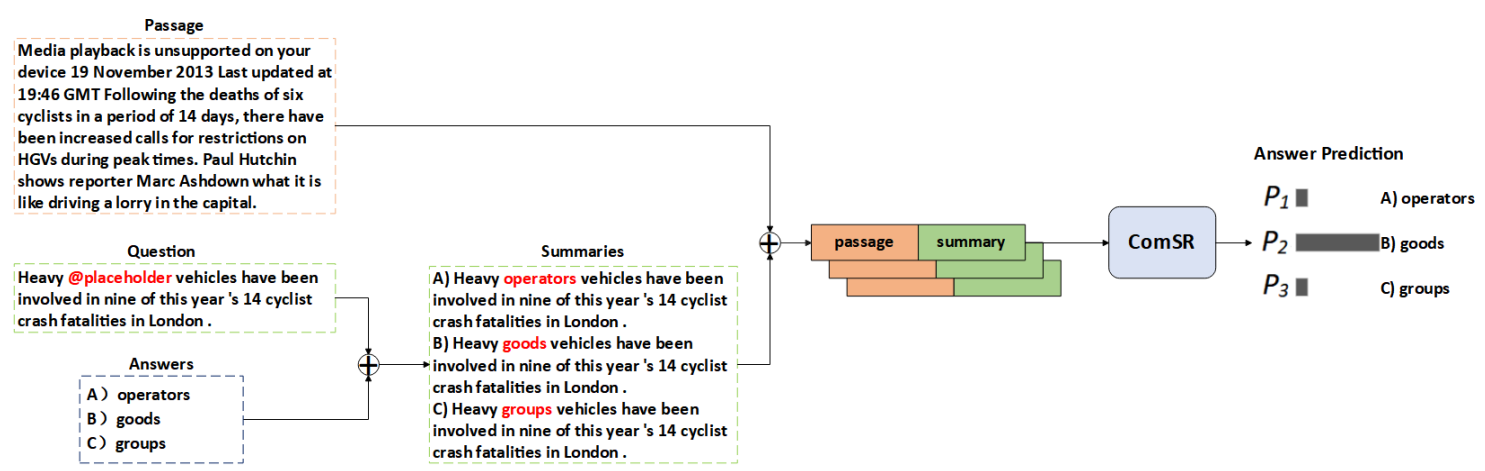

Figure 2: The framework of our model. By replacing the answer option into the question placeholder, answer obtains the context information from question, so the ambiguity is eliminated.

sage, we input passage and summary together to better reflect the corresponding relationship between the passage and the summaries. As BERT has been shown to be a powerful feature extractor for various tasks(Ran et al., 2019), we use BERT as the encoder to extract the vector representation of the semantic meaning of the passage and the summaries, as well as the relationship between the passage and the summaries. Finally, we use a classifier layer to convert the hidden state into the final selection of the model.

\subsection{Input Layer}

The initial question uses "@ placeholder" to represent the abstract word, which makes the question sentence not a complete sentence. We replace the "@ placeholder" in question with the answer word to get the summary $S$. As a complete sentence, $S$ provides rich context information to answer, thus effectively eliminating the ambiguity caused by polysemy in answer. Then the passage $P$ and the summary $S$ are concatenated, using [CLS] at the beginning and $[\mathrm{SEP}]$ at the end of sentences to get input vector.

$$
x=[p ; s]
$$

where $x$ denotes input vector, $p$ denotes passage vector and $s$ denotes summary vector.

\subsection{Encoder}

Although Pre-trained language models have achieved good results in various NLP tasks, most existing MCQA datasets are small in size, pretrained language models are hard to get adequate training. To solve this problem, we use the encoder obtained through multi-stage multi-task learning in MMM (Jin et al., 2020) as the initial encoder ${ }^{1}$. The reasons are as follows. First, the use of multiple data sets for training makes up for the shortcomings of the small amount of data in a single data set. It uses four similar task data sets, MultiNLI(Williams et al., 2017), SNLI (Young et al., 2014), DREAM (Sun et al., 2019), and RACE (Lai et al., 2017) for training, which has significantly more data than a single MCQA data set, thus solving the defect of insufficient data in a single data set. Second, the model uses two natural language inference datasets, MNLI and SNLI, as out-of-domain

\footnotetext{
${ }^{1}$ https://drive.google.com/ drive/folders/1EECS9na9PpX9CO_ cCzYj9FDkiBvOpyxv
} 
source datasets for Coarse-tuning, and two MCQA datasets, DREAM and RACE, as in-domain source datasets for Multi-task learning. The characteristics of different data sets make the model have better generalization ability(Jin et al., 2020). Third, because these data sets are highly related to ReCAM, it is suitable to use MMM encoder as initial encoder of ComSR.

$$
\mathbf{H}^{p}=\operatorname{Bert}(\mathbf{p}), \quad \mathbf{H}^{s}=\operatorname{Bert}(\mathbf{s})
$$

where $\operatorname{Bert}(\cdot)$ indicate BERT model which return the last attention layer. $\mathbf{H}^{p} \in R^{l \times|p|}, \mathbf{H}^{s} \in$ $R^{l \times|s|}$ are sequence representation of the passage and summary. $|p|,|s|$ are the length of the passage and summary. $l$ is the dimension of the hidden state.

\subsection{Answer Prediction}

In the answer prediction module, we pass the output of the encoder through a fully connected layer and a pooling layer to finally get the score of each option.

$$
s_{k}=\mathbf{v}_{s}^{T} \tanh \left(\mathbf{W}_{\mathbf{k}}\left[\mathbf{H}^{p} ; \mathbf{H}^{s}\right]+\mathbf{b}_{\mathbf{k}}\right)
$$

where $s_{k}$ is score of the answer, $\mathbf{v}_{s}^{T}$ and $\mathbf{W}_{\mathbf{k}}$ are learnable parameters. $\tanh (\cdot)$ performs tanh activation function, $\mathbf{W}_{k} \in \mathbf{R}^{l \times l}, \mathbf{b}_{k} \in \mathbf{R}^{l}, \mathbf{v}_{s}^{T} \in \mathbf{R}^{l}$.

The probability $P(k \mid P, S)$ of summary $S_{k}$ to be the correct answer is computed as

$$
P(k \mid P, S)=\frac{\exp \left(s_{k}\right)}{\sum_{i}^{N=5} \exp \left(s_{i}\right)}
$$

Then according to $P(k \mid P, S)$, the loss function is defined as

$$
J(\theta)=-\log (P(k \mid P, S))
$$

\section{Experimental setup}

\subsection{Dataset}

The data set of Reading Comprehension of Abstract Meaning (ReCAM) is provided by the organizer of the competition containing a large number of abstract words in answers. Abstract words refer to thoughts and concepts that are far from immediate perception. In ReCAM, we divide abstract words into Imperceptibility words and Nonspecificity words, and provide two data sets, respectively. The accuracy we showed in the paper was tested on the dev dataset.

\subsubsection{ReCAM-Imperceptibility}

Imperceptible words are the words of being Imperceptibility by eyes or senses, such as experience, success, significant, challenge. This data set provides a passage, a question with a placeholder and five answers with an imperceptibility word. The training set/test set/validation set contains 3227/2025/837 pieces of data respectively.

\subsubsection{ReCAM-Nonspecificity}

Nonspecific words are words with very broad concepts, and hypernyms are often words of this type, such as food, jewelry, people, and vehicle. This data set provides a passage, a question with a placeholder and five answers with a Nonspecificity word. The training set/test set/validation set contains 3318/2017/851 pieces of data respectively.

\subsection{Implementation Details}

In the test method, we use the accuracy of the model in the answer option of the data set as the measurement standard. For model training, we will train each tested model for 10 epochs at a learning rate of $3 e-05$, use cross-entropy to calculate loss, and use Adam optimizer(Kingman and $\mathrm{Ba}$, 2015) for fine-tuning. On hardware devices, we use GeForce RTX-2080Ti to provide computing.

\section{Results}

\subsection{Comparison with Baselines}

Since our model uses BERT as encoder, we use the original BERT(Devlin et al., 2018) for comparison. As pre-trained language models like BERT, we use ALBERT(Lan et al., 2019) and RoBERTa(Liu et al., 2019) in the experiment. In addition, we also adopt MMM(Jin et al., 2020) that performed well in multi-choice data sets such as RACE(Lai et al., 2017) and DREAM(Sun et al., 2019) to do experiments.

\begin{tabular}{lll}
\hline Model & \multicolumn{2}{l}{ Subtask } \\
& Subtask \\
& 2(\%) \\
\hline BERT-base & 47.19 & 50.65 \\
RoBERTa & 22.34 & 22.44 \\
ALBERT & 36.91 & 37.25 \\
MMM+BERT-base & 57.11 & 59.11 \\
ComSR+BERT-base(ours) & $\mathbf{6 4 . 7 6}$ & $\mathbf{6 4 . 8 6}$ \\
\hline
\end{tabular}

Table 1: Experimental results on ReCAM. The overall best results are in bold face. 


\begin{tabular}{lll}
\hline Model & $\mathbf{I} \rightarrow \mathbf{N}(\%)$ & $\mathbf{N} \rightarrow \mathbf{I}(\%)$ \\
\hline ComSR(Passage + summary)(ours) & $50.65(14.11 \downarrow)$ & $51.73(13.13 \downarrow)$ \\
ComSR(Passage + summary + question) & $48.53(17.18 \downarrow)$ & $49.94(15.51 \downarrow)$ \\
ComSR(Passage + summary + answer) & $44.89(18.55 \downarrow)$ & $46.71(18.38 \downarrow)$ \\
MMM(Passage + question + answer) & $39.48(17.63 \downarrow)$ & $43.73(15.38 \downarrow)$ \\
\hline
\end{tabular}

Table 2: Comparison generalization on ReCAM. I $\rightarrow \mathrm{N}$ denotes training on the Imperceptibility dataset and testing on the Nonspecificity dataset, $\mathrm{N} \rightarrow \mathrm{I}$ is the opposite. $\downarrow$ represents the drop for a model compared to the test in its own test set and + denotes concatenation.

Table 1 show that the model has similar features in task 1 and task2. In the experimental results of task1 and task2, we can find that (1) Although pre-trained language models have slightly different results depending on the models, the overall level is low on the small data set. (2) MMM proposed for the MCQA task performs better than the pre-trained language model on ReCAM. This is because the encoder of MMM uses the Multi-stage Multi-task Learning to train on NLI data sets such as MNLI and SNLI and on MCQA data sets such as DREAM and RACE, which is more suitable for ReCAM. (3) The performance of ComSR is better than other baselines, and it has significantly improved. It can be seen that, under the condition of using the same encoder, replacing the placeholder of question with answer is more conducive to understand sentence meaning.

\subsection{Analysis Studies}

\subsubsection{The completeness of the semantic expression of summary}

Converting question and answer into summary input improves semantic integrity and the model's ability to understand sentences. However, is the semantics provided by summary complete? To study this problem, we set up the following experiment.

\begin{tabular}{lll}
\hline Model & $\begin{array}{l}\text { Subtask } \\
\mathbf{1}(\%)\end{array}$ & $\begin{array}{l}\text { Subtask } \\
\mathbf{2}(\%)\end{array}$ \\
\hline $\begin{array}{l}\text { ComSR(Passage }+64.76 \\
\text { summary)(ours) }\end{array}$ & 64.86 \\
$\begin{array}{l}\text { ComSR(Passage }+65.71 \\
\text { summary + ques- } \\
\text { tion) }\end{array}$ & 65.45 \\
$\begin{array}{l}\text { ComSR(Passage + 63.44 } \\
\text { summary + answer) }\end{array}$ & 65.09 \\
\hline
\end{tabular}

Table 3: Comparison among different implementation of the input method on ReCAM. + denotes concatenation.
If there is a loss of semantics in the synthesis of the summary, then the summary can be supplemented when the original question and answer are used, thereby improving the performance of the model. However, it can be seen from the table 3 results that some of the experimental results of the passage+ summary+answer and passage+summary+question groups are equal to passage+summry and even slightly lower than passage+summary. Therefore, the conversion of question and answer into summary makes the semantics of options more complete, and there is no semantic loss.

\subsubsection{Research on model generalization}

To study the generalization ability of the model, (1) we use the ReCAM-Imperceptibility data set to train and test it in the ReCAM-Nonspecificity data set. (2) we use the ReCAM-Nonspecificity data set to train and test it in the ReCAM-Imperceptibility data set. In order to study the most suitable input form, we use passage+summary+question, passage+summary+answer and MMM (passage+question+answer) model for comparison.

According to the experimental results in table 2, after switching the data set for testing, the highest accuracy is ComSR(Passage + summary). Although the results of the test in another data set have dropped, the ComSR using the Passage and summary received the least impact. In summary, ComSR(Passage + summary) performs best in the above models, and the method of matching summary and Passage alone has stronger generalization ability.

\subsubsection{Case Analysis}

Table 4 shows a passage and its corresponding question and answers. To understand ComSR's ability to disambiguate, we specifically selected samples that contained polysemous words in answers. In this example, the answer "goods" is a typical polysemous word. 


\begin{tabular}{|l|l|}
\hline Passage & $\begin{array}{l}\text { Media playback is unsupported } \\
\text { on your device 19 November } \\
\text { 2013 Last updated at 19:46 GMT } \\
\text { Following the deaths of six cy- } \\
\text { clists in a period of 14 days, there } \\
\text { have been increased calls for re- } \\
\text { strictions on HGVs during peak } \\
\text { times. Paul Hutchin shows re- } \\
\text { porter Marc Ashdown what it is } \\
\text { like driving a lorry in the capital. }\end{array}$ \\
\hline Question & $\begin{array}{l}\text { Heavy @ placeholder vehicles } \\
\text { have been involved in nine of this } \\
\text { year 's 14 cyclist crash fatalities } \\
\text { in London. }\end{array}$ \\
\hline Answers & $\begin{array}{l}\text { (a) operators (b) goods (c) } \\
\text { groups (d) patrol (e) air }\end{array}$ \\
\hline
\end{tabular}

Table 4: Example of ReCAM.

Models take the logarithm of the value to get the predictions. Because many of the predictions are negative, we add bias $=12.5$ to the predictions for easy viewing and show it in Figure 3.

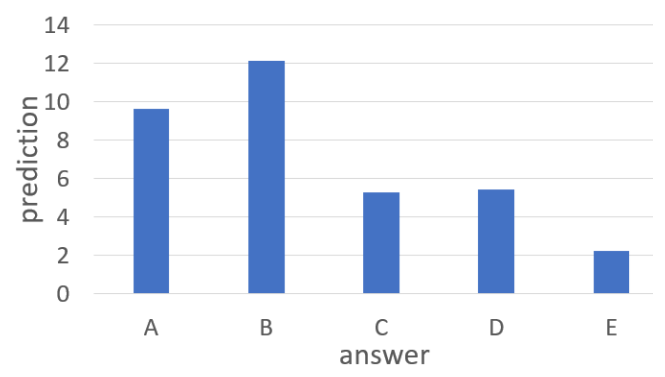

(a) MMM(passage + question + answer)

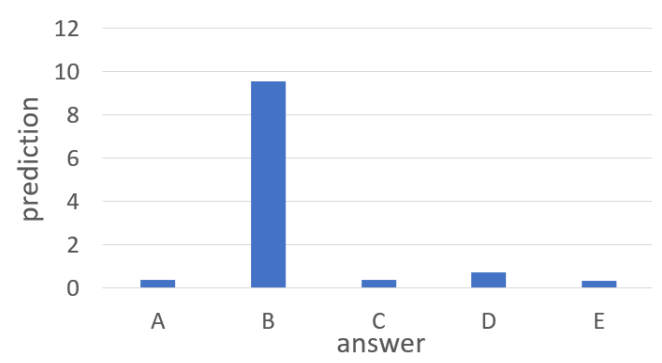

(b) ComSR(passage + summary)

Figure 3: Prediction distributions in different models. The model selects the answer with the highest predicted value as the output option.

We observe that although MMM selects the right option, the difference between the correct answer and other option values in the probability distribution is not obvious. On the contrary, correct prediction value is much higher than other values in ComSR. By combining question and answer into summary, ComSR can not only improve the semantic integrity of the sentence, but also eliminate the ambiguity caused by the polysemy of the original answer. Therefore, even for polysemous words that are prone to ambiguity, ComSR can obtain accurate answers by fully understanding the summary representation.

\section{Conclusion}

We proposed ComSR for multiple choice reading comprehension to complete summary representation. Merging the separated question and answer into a summary could improve semantic integrity and better reveal the relationship between option and question. Results showed that our model can achieve relatively high performance compared to the latest baseline in terms of accuracy and generalization ability.

\section{References}

Mark A Changizi. 2008. Economically organized hierarchies in wordnet and the oxford english dictionary. Cognitive Systems Research, 9(3):214-228.

Jacob Devlin, Ming-Wei Chang, Kenton Lee, and Kristina Toutanova. 2018. Bert: Pre-training of deep bidirectional transformers for language understanding. arXiv preprint arXiv:1810.04805.

Di Jin, Shuyang Gao, Jiun-Yu Kao, Tagyoung Chung, and Dilek Hakkani-tur. 2020. Mmm: Multi-stage multi-task learning for multi-choice reading comprehension. In Proceedings of the AAAI Conference on Artificial Intelligence, volume 34, pages 8010-8017.

DP Kingman and J Ba. 2015. Adam: A method for stochastic optimization. conference paper. In $3 r d$ International Conference for Learning Representations.

Guokun Lai, Qizhe Xie, Hanxiao Liu, Yiming Yang, and Eduard Hovy. 2017. Race: Large-scale reading comprehension dataset from examinations. arXiv preprint arXiv:1704.04683.

Zhenzhong Lan, Mingda Chen, Sebastian Goodman, Kevin Gimpel, Piyush Sharma, and Radu Soricut. 2019. Albert: A lite bert for self-supervised learning of language representations. arXiv preprint arXiv:1909.11942.

Yinhan Liu, Myle Ott, Naman Goyal, Jingfei Du, Mandar Joshi, Danqi Chen, Omer Levy, Mike Lewis, Luke Zettlemoyer, and Veselin Stoyanov. 2019. Roberta: A robustly optimized bert pretraining approach. arXiv preprint arXiv:1907.11692. 
Qiu Ran, Peng Li, Weiwei $\mathrm{Hu}$, and Jie Zhou. 2019. Option comparison network for multiplechoice reading comprehension. arXiv preprint arXiv:1903.03033.

Otfried Spreen and Rudolph W Schulz. 1966. Parameters of abstraction, meaningfulness, and pronunciability for 329 nouns. Journal of Verbal Learning and Verbal Behavior, 5(5):459-468.

Kai Sun, Dian Yu, Jianshu Chen, Dong Yu, Yejin Choi, and Claire Cardie. 2019. Dream: A challenge data set and models for dialogue-based reading comprehension. Transactions of the Association for Computational Linguistics, 7:217-231.

Adina Williams, Nikita Nangia, and Samuel R Bowman. 2017. A broad-coverage challenge corpus for sentence understanding through inference. arXiv preprint arXiv: 1704.05426 .

Peter Young, Alice Lai, Micah Hodosh, and Julia Hockenmaier. 2014. From image descriptions to visual denotations: New similarity metrics for semantic inference over event descriptions. Transactions of the Association for Computational Linguistics, 2:67-78.

Boyuan Zheng, Xiaoyu Yang, Yu-Ping Ruan, Quan Liu, Zhen-Hua Ling, Si Wei, and Xiaodan Zhu. 2021. Semeval-2021 task 4: Reading comprehension of abstract meaning.

Pengfei Zhu, Hai Zhao, and Xiaoguang Li. 2020. Dual multi-head co-attention for multi-choice reading comprehension. arXiv preprint arXiv:2001.09415. 